\title{
Security System for the Telecommunication Room Based on the RFID and Video Analysis
}

\author{
Gongyin Luo ${ }^{1}$, Junfeng Dai $^{1}$, Bin Yang ${ }^{1}$, Xingxing Luo ${ }^{2, *}$ and Lixian Chen ${ }^{2}$ \\ ${ }^{1}$ Information \& communication, State grid information \& communication Branch of Hubei EPC, Hubei, China, 430077 \\ ${ }^{2}$ Information Technology, Xiamen Great Power Geo Information Technology Co., Ltd Xiamen, China 361009 \\ ${ }^{*}$ Corresponding author
}

\begin{abstract}
Considering the importance of the data and equipment in the telecommunication room, it is necessary to ensure the security of the telecommunication room and prevent the intruders in the early time. In this paper, we proposed a security system based on the RFID and the video analysis. The information of the people who are allowed to enter the telecommunication room is collected and stored in electronic RFID tags. The people who want to enter the room are identified by the entrance system, and tracked by the RFID reader from the antenna. However, the entrance system cannot prevent the intruder rigorous, and meanwhile, once the people are out of the range of the RFID, the video surveillance will be launched to track the RFID tags. The proposed method can improve the efficiency of the security management and decrease the labor force and cost. The experiment results show the $100 \%$ detection accuracy for identifying the intruders and tracking the RFID tags.
\end{abstract}

Keywords-RFID; security; surveillance

\section{INTRODUCTION}

Radio frequency identification (RFID) [1-3] is a genetic term that is used to describe a system that transmits the identity in the form of a unique serial number of an object or person wirelessly based on the radio waves. RFID can recognize target and obtain the related information automatically. RFID consists of reader, data accumulator and electronic tags. The reader works as a transceiver or an interrogator and emits electronic signals to the RFID tags by the radio waves. By scanning the RFID tags [4], the data stored in the tags are read and retrieved, and then sent to the data accumulator. The electronic tags are used to store the related information [5]. Figure 1 shows the components of the RFID.

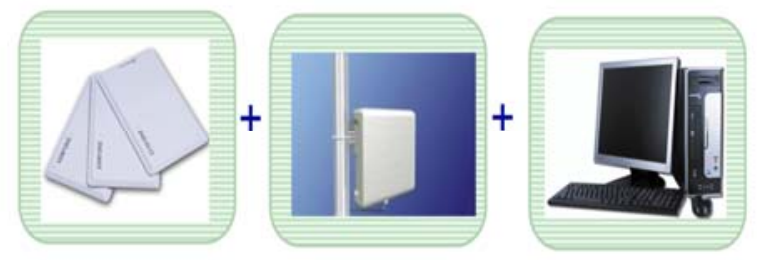

FIGURE I. COMPONENTS OF THE RFID (RFID TAG + READER + DATA ACCUMULATOR)

The entrance and tracking system [6] is mainly used in intelligent building for the security of the enterprise and the government departments. Entrance systems of residential units mainly prevent from theft. In this paper, we employ the entrance system and tracking system based on the RFID and the video analysis for the security of the telecommunication room.

\section{RFID-BASED IDENTIFICATION}

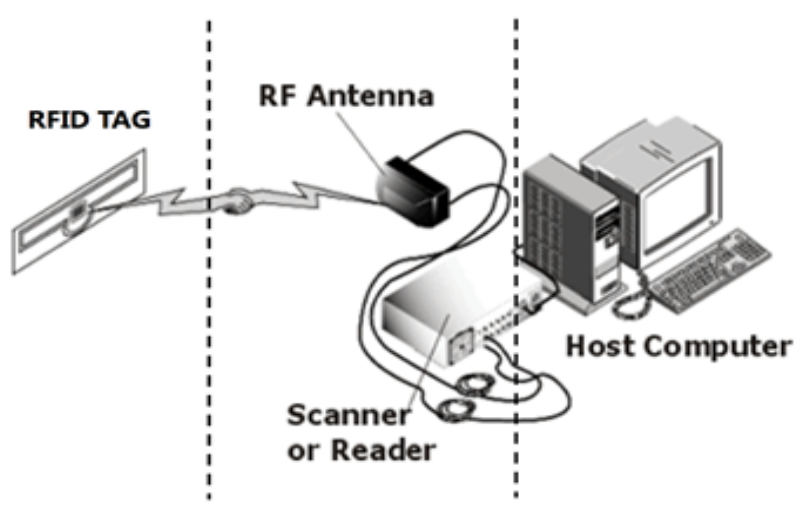

FIGURE II. THE STRUCTURE OF THE RFID

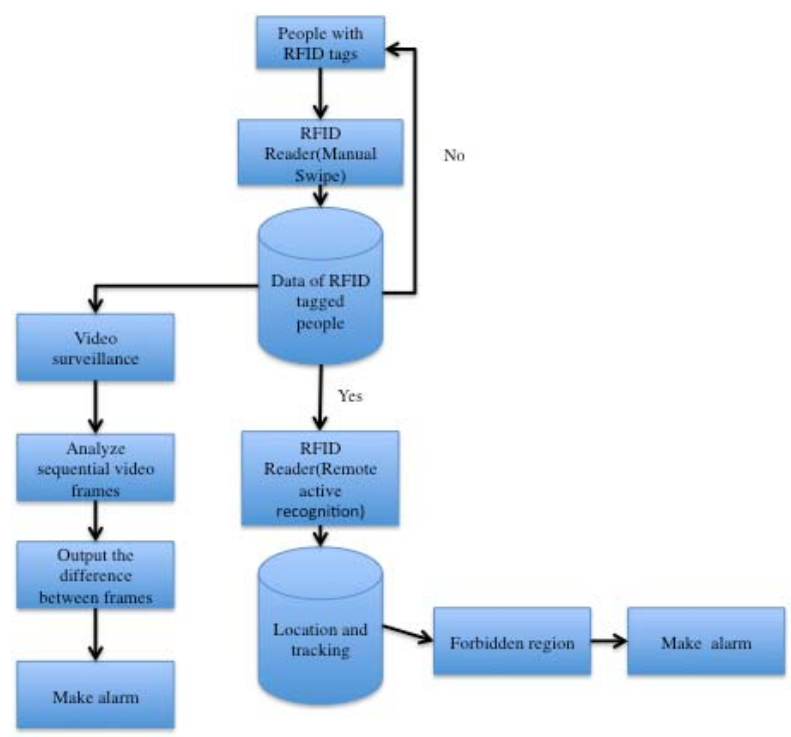

FIGURE III. SYSTEM DIAGRAM OF THE PROPOSED SECURITY SYSTEM BY COMBINING VIDEO CAMERA AND RFID SENSORS

The structure of the RFID is shown in Figure 2. Figure 3 shows the diagram of the proposed security system by 
combining video camera and RFID. First, the person who wants to enter the telecommunication room are supposed get a red RFID tag, which is used store the related information about the corresponding person. Then by swiping the RFID tags, the person can get the authority to enter the room.

Then, the RFID reader that has the property of remote active recognition is launched to track the legal object or person. Once the person enters the forbidden region, the system makes alarm to the specific device or department. We employed the RFID reader that works in a radio frequency of 2.2-2.4GHZ and can identify the object within 50 meters. The experiment shows that our RFID-based method achieves an accuracy rate of $100 \%$ for identifying and tracking the target object.

\section{VIDEO ANALYSIS FOR PREVENTING THE INTRUDER}

For the entrance system, although the RFID reader can identify the object accurately, but the entrance system cannot prevent from the intruder rigorously. For example, once a person swipes the card, more people can enter the telecommunication room in theory. Therefore, we employed the video analysis for the entrance system to provide more rigorous surveillance. We proposed a method using a video camera to monitor the target. The background image is updated adaptively. When the RFID reader of the entrance system gets one object's information, which means that new object wants to enter in, the background model stops from updating. We selected the area of $5 * 5$ meters inside the door as the monitor area. The number of object entering the room in one moment is obtained by similar techniques used in face recognition [7, 8] and pathological brain detection [9-11].

For the tracking system, the objects must be in range of the RFID antenna. In order to save the cost of the device, if there is just a small region out of the range of RFID, we proposed a system to continue to track the target based on RFID when the object is out of range. The vision analysis system consists of 4 main components: (a) modeling the background, (b) identifying moving objects based on the background subtraction, (c) segmenting the objects using color and shape model, which are described in fruit-classification [12-14]; and (d) tracking moving objects.

In the tracking system, we took the background model adaptively updated. Once a new person enter specific region which is out of the range of the RFID antenna, the background image is prevented from updating until all people get out of the small region. In the system, we used the tag's color and shape information to segment the RFID tags from the other moving object.

\section{EXPERIMENT}

To evaluate the performance of the proposed system, we conducted two tests for the entrance and tracking system respectively.

Test 1: One people swiped the card, another person followed him to enter in.
Test 2: One people entered the small region, which is out of range of antenna.

In the first test, the system is accomplished via the face recognition. We utilized the algorithm based on MSNN [15, 16] to get the number the people who want to enter in. Figure 4 (a) shows an image from the video camera, and Figure 4 (b) shows an example of the detection result. The detection result means that the monitor system gets the number of people who want to enter is 2 , however, the RFID reader gets just one piece of related information. Thus, the exception is fed back to the specific people or department to make alarm. In order to test the accuracy of the first test, we get 10 people, and enter the room in random set. The detection result shows that our method achieved the accuracy of $100 \%$.

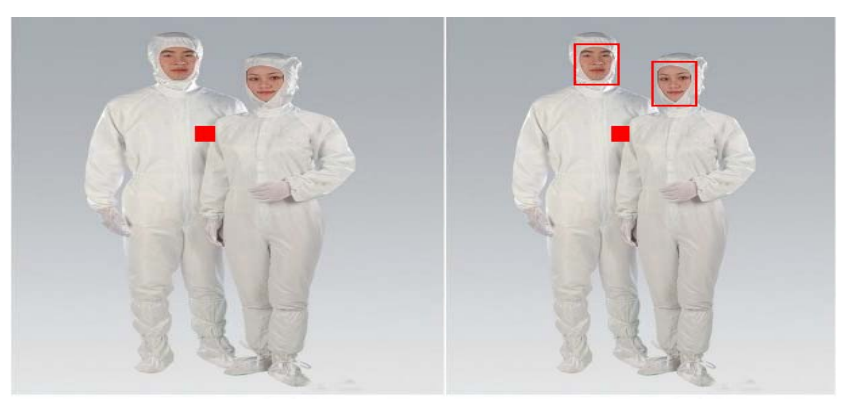

(a)

(b)

FIGURE IV. (A) ORIGINAL IMAGE (B) DETECTION RESULT

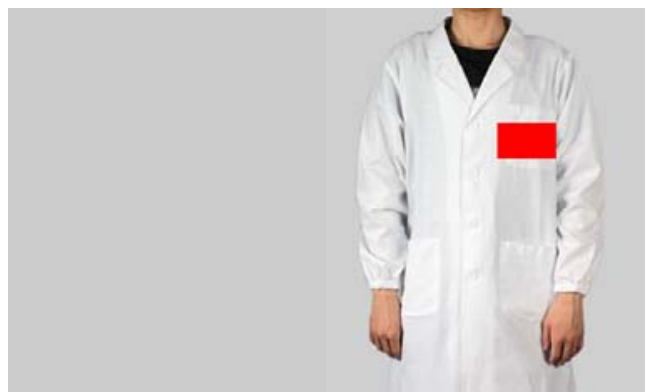

(a)

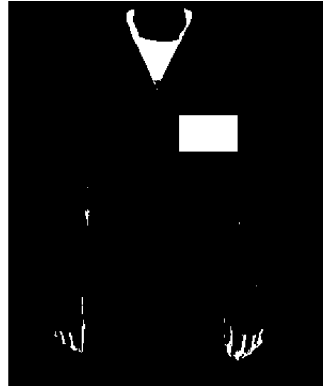

(C) (b)

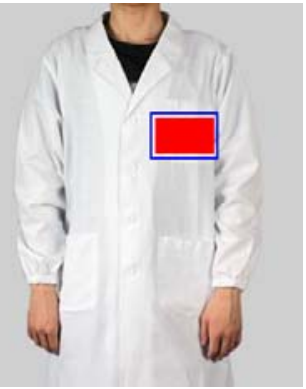

(d)
FIGURE V. (A) THE BACKGROUND, (B) ONE FRAME OF THE VIDEO CAMERA, (C) SUBTRACTION (A) FROM (B), (D) MARK OF THE RFID

In the second test, we requested all the workers to wear the red RFID tags. All people who enter the telecommunication must wear the Antistatic clothing in white color. Figure 5 shows the result of RFID detection result. Figure 5 (a) is the background image. Figure 5 (b) is one frame of the video selected randomly. Figure 5 (c) shows the subtraction result of 
Figure 5 (b) from Figure 5 (a), and Figure 5 (d) shows the final detection result of the RFID tag, which can be further recognized by the RDIF reader. In order to test the detection accuracy, we select 50 frames from the video camera, in which, 35 frames contain RFID tags, and 15 frames do not contain RFID tags. The detection result shows that our proposed algorithm can get $100 \%$ accuracy.

The resolution of images is $320 \times 240$ pixels. The average speed of the RFID-based and vision-based algorithms for detecting the moving people and his RFID tag is 0.7042 seconds and 0.02413 seconds respectively. The accuracy rate of identifying and tracking the target people is $100 \%$ in testing with the 10 people. Figure 6 demonstrates the example results of identification and tracking of people who are out of the range of RFID antenna.

\section{CONCLUSION AND FUTURE WORK}

In this paper, we proposed a security and tracking system based on the RFID and video analysis. The experiment shows that our method performs well.

Our future work will focus extending the application and try to save the cost as much as possible. The video analysis method will be applied in Alzheimer's disease detection [1719], and tea-category classification [20].

\section{ACKNOWLEDGEMENT}

This work was supported by JiAo foundation (S11180BY1501).

\section{REFERENCE}

[1] Hossain, M. A. and M. Quaddus, "Radio frequency identification (RFID) adoption: A cross-sectional comparison of voluntary and mandatory contexts," Information Systems Frontiers, Vol. 17, No. 5, pp. 1057-1076, Oct 2015.

[2] Guo, D., et al., "Improved Radio Frequency Identification Indoor Localization Method via Radial Basis Function Neural Network," Mathematical Problems in Engineering, No. pp. 2014.

[3] Hassouni, S. and H. Qjidaa, "A design of analog VDD generator for passive UHF RFID Tag in $90 \mathrm{~nm}$ CMOS," International Journal of Microwave and Wireless Technologies, Vol. 7, No. 5, pp. 507-513, Oct 2015.

[4] Cortes, F. P., et al., "A power management system architecture for LF passive RFID tags," Analog Integrated Circuits and Signal Processing, Vol. 85, No. 1, pp. 47-55, Oct 2015.

[5] Hervert-Escobar, L., et al., "Methods of selection and identification of RFID tags," International Journal of Machine Learning and Cybernetics, Vol. 6, No. 5, pp. 847-857, Oct 2015.

[6] Min, D. G., J. W. Kim, and M. S. Jun, "The Entrance Authentication and Tracking Systems Using Object Extraction and the RFID Tag," in Ubiquitous Computing and Multimedia Applications, Pt I. vol. 150, T. H. Kim, et al., Eds., ed Berlin: Springer-Verlag Berlin, 2011, pp. 313-326.

[7] Siddiqi, M. H., et al., "Facial expression recognition using active contour-based face detection, facial movement-based feature extraction, and non-linear feature selection," Multimedia Systems, Vol. 21, No. 6, pp. 541-555, Nov 2015.

[8] Hennion, S., et al., "Characterization and prediction of the recognition of emotional faces and emotional bursts in temporal lobe epilepsy," Journal of Clinical and Experimental Neuropsychology, Vol. 37, No. 9, pp. 931945, Oct 2015

[9] Zhang, Y., et al., "Pathological brain detection in magnetic resonance imaging scanning by wavelet entropy and hybridization of biogeography-based optimization and particle swarm optimization," Progress In Electromagnetics Research, Vol. 152, No. pp. 41-58, 2015.

[10] Zhang, Y., et al., "Pathological brain detection based on wavelet entropy and $\mathrm{Hu}$ moment invariants," Bio-Medical Materials and Engineering, Vol. 26, No. s1, pp. 1283-1290, 2015.

[11] Wang, S., et al., "Feed-forward neural network optimized by hybridization of PSO and ABC for abnormal brain detection," International Journal of Imaging Systems and Technology, Vol. 25, No. 2, pp. 153-164, 2015.

[12] Wang, S., et al., "Fruit Classification by Wavelet-Entropy and Feedforward Neural Network Trained by Fitness-Scaled Chaotic ABC and Biogeography-Based Optimization," Entropy, Vol. 17, No. 8, pp. 5711-5728, 2015.

[13] Zhang, Y., et al., "Fruit classification using computer vision and feedforward neural network," Journal of Food Engineering, Vol. 143, No. 0, pp. 167-177, 2014.

[14] Wu, L. and Y. Zhang, "Classification of Fruits Using Computer Vision and a Multiclass Support Vector Machine," Sensors, Vol. 12, No. 9, pp. 12489-12505, 2012.

[15] Guo, Y. Y. and L. H. Jiang, Morphological shared-weight probabilistic neural networks for pattern classification of SAR images. New York: Ieee, 2007.

[16] Ke, C., White blood cell detection using a novel fuzzy Morphological shared-weight Neural Network. Los Alamitos: Ieee Computer Soc, 2008.

[17] Aubry, S., et al., "Assembly and Interrogation of Alzheimer's Disease Genetic Networks Reveal Novel Regulators of Progression," Plos One, Vol. 10, No. 3, pp. 25, Mar 2015.

[18] Zhang, Y., et al., "Detection of subjects and brain regions related to Alzheimer's disease using 3D MRI scans based on eigenbrain and machine learning," Frontiers in Computational Neuroscience, Vol. 66, No. 9, pp. 1-15, 2015.

[19] Zhang, Y., et al., "Detection of Alzheimer's disease and mild cognitive impairment based on structural volumetric MR images using 3D-DWT and WTA-KSVM trained by PSOTVAC," Biomedical Signal Processing and Control, Vol. 21, No. pp. 58-73, 8// 2015.

[20] Wang, S., et al., "Identification of Green, Oolong and Black Teas in China via Wavelet Packet Entropy and Fuzzy Support Vector Machine," Entropy, Vol. 17, No. 10, pp. 6663-6682, 2015. 